Isaree Teriyapirom, Andreia S. Batista-Rocha und Bon-Kyoung Koo

\title{
3.3 Genetic Engineering von Organoiden ${ }^{10}$
}

Zusammengefasst und aus dem Englischen übersetzt von Lilian Marx-Stölting, Sandra Pilat-Carotta und Aileen-Diane Bamford

Seit ihrer Entwicklung haben sich Organoide rasch zu einem beliebten Modell zur Erforschung von Krankheiten sowie von Aufbau und Reparatur von Organen etabliert (siehe auch Einleitung, Kap. 2.1). 3-D-Organoide stellen ein wesentlich physiologischeres Modell als die bisher hauptsächlich verwendete zweidimensionale Zellkultur dar, und sind gleichzeitig weniger zeitaufwendig und ethisch vertretbarer als Tiermodelle. Um die Entwicklung von Krankheiten wie Krebs besser studieren und nachvollziehen zu können, werden Organoide genetisch verändert. Dabei werden spezifische Genmutationen in Organoide eingebracht und Genomsequenzen repariert, verändert oder blockiert. Dadurch ermöglichen so veränderte Organoide neben der Erforschung von Krankheiten auch Screenings des gesamten Genoms und die Generierung von Reporterorganoiden, ${ }^{11}$ bis hin zur personalisierten Medizin. Somit etabliert sich gerade ein neues Forschungsfeld mit enormer Bedeutung sowohl für die Grundlagenforschung als auch für die angewandte klinische Forschung: die Organoidgenetik („organoid genetics").

Es gibt derzeit verschiedene Methoden des Genetic Engineering, die in Organoiden verwendet werden können, um eine spezifische Veränderung von DNA-Sequenzen zu erzielen. Wenn solche Änderungen in einer ein Protein kodierenden Sequenz erfolgen, können sie dieses Protein gezielt verändern und in Folge Aufschluss über dessen biologische Funktion in der Zelle oder im Zellverband geben. Dazu müssen die genetischen

10 Dieser Beitrag ist eine deutsche Zusammenfassung des Review-Artikels „Genetic engineering in organoids" von Isaree Teriyapirom, Andreia S. Batista-Rocha und Bon-Kyoung Koo, der $2020 \mathrm{im}$ Journal of Molecular Medicine erschienen ist und eine umfangreiche Darstellung des wissenschaftlichen Sachstands sowie der aktuellen Literatur zum Thema (Stand Frühjahr 2020) bietet.

11 Reporterorganoide erlauben es, mittels eingefügten Fluoreszenzproteinen oder Enzymen, die ein sichtbares oder messbares Signal erzeugen, die An- oder Abschaltung des gewünschten Gens zu verfolgen. 
Werkzeuge jedoch erst einmal in die Zielzellen eingeschleust werden; dieser Vorgang wird als „Delivery“ bezeichnet.

\subsubsection{Delivery-Methoden}

In der Organoidforschung wurden bislang vor allem zwei Methoden zur Einführung genetischer Komponenten in Organoide eingesetzt: virale und nicht-virale Methoden (siehe Abbildung 1). Beide Vorgehensweisen haben Vor- und Nachteile, sodass bei der Wahl der Methode die Eigenschaften der Zielzelle, die Größe des DNA-Fragments sowie die nötige Dauer der Genexpression berücksichtigt werden müssen.

Bei den viralen Methoden werden bestimmte Viren (etwa Retroviren, Lentiviren oder Adenoviren) gentechnisch soweit verändert, dass sie eine künstlich eingefügte DNA- oder RNA-Sequenz ${ }^{12}$ in die Zellen einschleusen, gleichzeitig aber ihre Fähigkeit behalten, Zellen zu infizieren. Bei der Verwendung von Retro- und Lentiviren kommt eine stabile Integration der fremden Gensequenzen ins Genom zustande, wodurch die Mutation zuverlässig auch an die Nachkommen der Zelle vererbt wird. Retroviren sind auf Hilfe durch den Zellzyklus der Wirtszelle angewiesen, um ihre genetische Information stabil in das Genom integrieren zu können, weshalb sie keine terminal differenzierten, sich nicht mehr teilende Zellen infizieren können. Außerdem wird eine relativ große Menge an Viren für eine Infektion mit Retroviren benötigt, was zu einer Immunreaktion des Wirts führen kann, die die Effizienz der Genomintegration mitunter verringert. Auf Lentiviren treffen diese Einschränkungen nicht zu, weshalb sie häufig genutzt werden, um Mutationen in Immunzellen oder Zellen, die sich nicht mehr teilen, zu bringen. Allerdings erfolgt die Integration sowohl bei Retro- als auch bei Lentiviren bevorzugt an transkriptionell aktiven Stellen im Genom, an denen vermehrt Gene abgelesen werden, was wiederum die Genexpression der Wirtsgene negativ beeinflussen kann. Darüber hinaus können beide viralen Vektoren nur DNA-Abschnitte übertragen, die eine bestimmte Größe (8kb/8000 Basen) nicht überschreiten: Dies ist für viele Experimente ausreichend, aber nicht für alle.

Die adenovirale Methode umgeht die dauerhafte Integration der Fremd-DNA, indem die eingebrachte Erbinformation in der Zielzelle als Episom (ein zusätzlich zu den Chromosomen vorliegendes ringförmiges DNA-Molekül) bestehen bleibt. Adenovirale

12 „RNA“ steht für Ribonucleic Acid (Ribonukleinsäure) und ist ein einsträngiges Makromolekül, welches im Zellkern und im Zytoplasma von Zellen vorkommt und eine wichtige Rolle bei der Proteinbiosynthese spielt, also bei der Umsetzung genetischer Informationen in Proteine, deren Bauanleitung die sogenannte mRNA (messenger-RNA oder Boten-RNA) liefert. Darüber hinaus können weitere RNAs auch an der Regulierung der Genexpression beteiligt sein. 
Abbildung 1: Methoden zur Erzeugung von Organoiden und Genetic Engineering mit ihren Anwendungsmöglichkeiten

Transposon-basiertes System

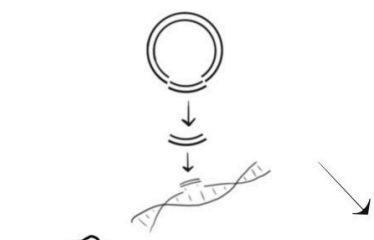

\section{CRISPR/Cas9-System}

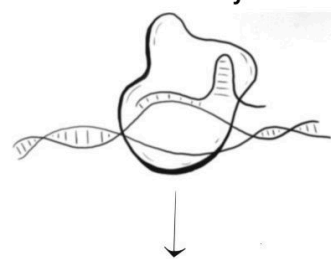

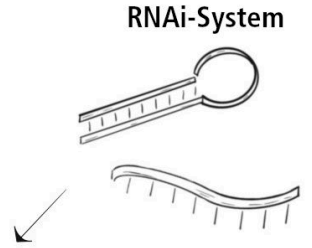

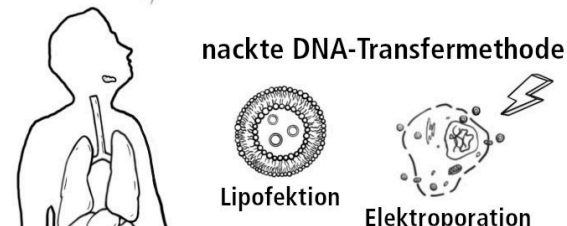

virale Methode

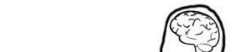

Elektroporation
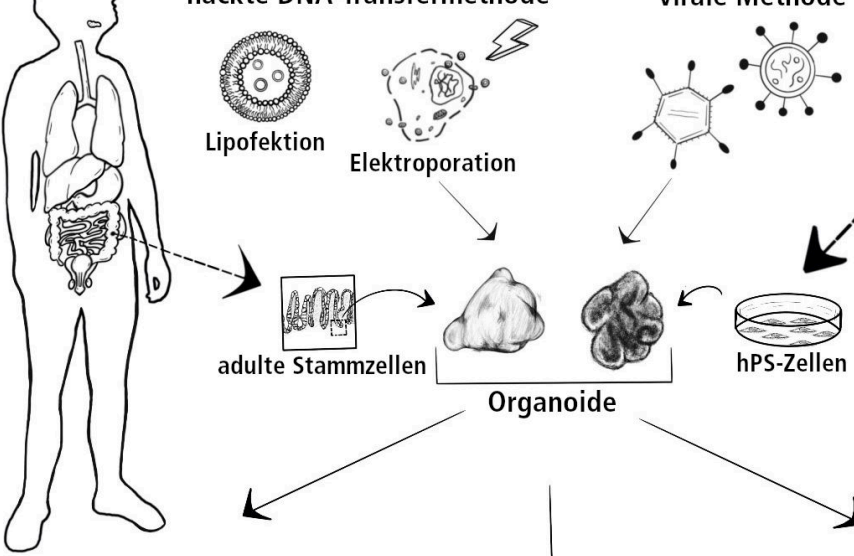
hPS-Zellen Organoide
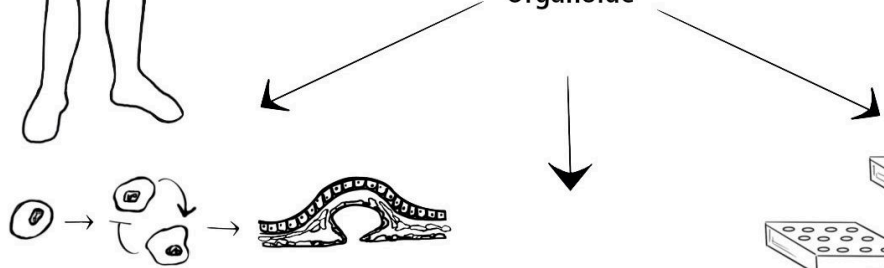

Regulation der Gewebeentwicklung

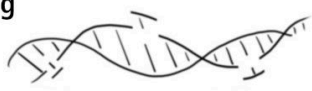

Drug Screenings

\section{Krankheitsmodellierung}

Organoide können entweder aus adulten Stammzellen oder pluripotenten Stammzellen (hPS-Zellen) erzeugt werden. Die verschiedenen Organe, die in den menschlichen Figuren dargestellt sind, repräsentieren die Arten von Organoiden, die mit adulten Stammzellen bzw. hPS-Zellen erzeugt wurden. Organoide können mit verschiedenen Genetic-EngineeringMethoden wie CRISPR/Cas9, Transposon-basierten Systemen oder RNA-Interferenz (RNAi) modifiziert werden. Diese Werkzeuge können den Organoiden mit nicht-viralen Ansätzen wie Lipofektion oder Elektroporation oder mit einem viralen Ansatz unter Verwendung von Retrovirus, Lentivirus oder Adenovirus zugeführt werden. Die genetisch modifizierten Organoide können für verschiedene Anwendungen weiter genutzt werden, z. B. für die Modellierung der biologischen Entwicklung und von Krankheiten und für das Screening von Medikamenten für die Präzisionsmedizin. 
Einbringung funktioniert sowohl in sich teilenden als auch in sich nicht teilenden Zellen. Allerdings kann die mangelnde Integration ins Genom auch dazu führen, dass das eingeführte Gen während der folgenden Zellteilungen wieder verloren geht. Die Expression eines auf diese Weise in ein erwachsenes Tier eingebrachten Transgens wurde nur zwischen 5 und maximal 20 Tagen nach der adenoviralen Infektion nachgewiesen.

Alternativ zu viralen Methoden kann auch sogenannte „nackte“ DNA ohne viralen Vektor verwendet werden, die in der Regel entweder durch Elektroporation oder Lipofektion in die Zellen eingebracht wird. Elektroporation verursacht durch elektrische Impulse vorübergehend kleine Öffnungen in der Zellmembran, durch die die Fremd-DNA eindringen kann. Diese Methode ist prinzipiell für jeden Zelltyp und sogar für lebendige Organismen geeignet und ermöglicht auch das Einbringen größerer DNA-Abschnitte. Allerdings benötigt man hierfür ein spezielles Gerät und viele Pilotversuche, um die optimalen Parameter für den Transfer zu identifizieren, da diese für jedes Gerät und jeden Zelltyp variieren können. Die Lipofektion hingegen nutzt Lipide oder ähnliche Moleküle, die Liposomen - kleine, mit Flüssigkeit gefüllte Vesikel - bilden, und darin DNA einkapseln. Die Liposomen können mit Zellmembranen verschmelzen und so die eingekapselte DNA in die Zelle einschleusen. Diese Methode ist relativ einfach und ebenfalls in vielen Zelltypen effizient einsetzbar. Die Expression der auf diese Weise eingebrachten Transgene ist in der Regel nur vorübergehend (transient), außerdem kann die Lipofektion das Überleben der Zellen beeinträchtigen.

\subsubsection{Werkzeuge für Genetic Engineering}

In der Organoidgenetik werden verschiedene Methoden genutzt, um Gene zu verändern, also Genetic Engineering zu erreichen. Dazu zählen RNA-Interferenz (RNAi), eine auf der Expression bestimmter RNAs beruhende Methode, die Verwendung von Retro-/Lentiviren, Transposons (springende Gene) und das CRISPR/Cas9-System ${ }^{13}$ (auch „Genschere“/Genomchirurgie). Jede Methode hat ihre Vor- und Nachteile, auf die hier nicht im Detail eingegangen werden kann. ${ }^{14}$ Der Fokus dieser Zusammenfassung

13 „CRISPR“ steht für: Clustered regularly interspaced short palindromic repeats. "Cas" steht für CRISPR-associated. Eine Beschreibung des Verfahrens folgt im Text.

14 Für mehr Informationen zu den Methoden, die bei Organoiden eingesetzt werden, siehe den Orignalbeitrag (Teriyapirom et al., 2020). Für eine Übersicht verschiedener Methoden des GenomeEditings siehe etwa Khan, S. H. (2019): Genome-editing technologies: Concept, pros, and cons of various genome-editing techniques and bioethical concerns for clinical application. In: Molecular Therapy Nucleic Acids 16: 326-334. 
liegt auf der RNAi-Methode, der CRISPR/Cas9-Methode, der Verwendung von Retround Lentiviren sowie Transposons. Das RNAi-System schleust kurze, synthetisierte RNA-Abschnitte in die Zelle ein, die dann ein komplementäres Paar mit der mRNA des Zielgenes bilden und so deren Abbau verursachen oder die Translation in ein funktionelles Protein verhindern. Diese Methode ist in allen Säugetierzellen effektiv, und benötigt keine vorausgehende genetische Manipulation. Die RNAi-Technologie kann allerdings nur für Knock-downs, also die Ausschaltung von Genen, verwendet werden. Transposons wie das „PiggyBac-System“ oder „Sleeping Beauty“ sind eine gute Wahl, um stabile Genexpression über einen längeren Zeitraum zu erreichen. Sie schneiden die gewünschte Gensequenz aus und kopieren sie in eine andere, zufällig ausgewählte hinein, was zu ungewünschten Nebeneffekten führen kann.

Seit 2012 wurden CRISPR/Cas-Systeme für sequenzspezifisches Editieren sowohl in prokaryotischen (Bakterien) als auch eukaryotischen Zellen adaptiert. Das System beruht auf zwei Komponenten, der Cas9-Endonuklease („Genschere“) und einer „guide RNA“ (gRNA), die Cas9 an eine spezifische Stelle im Zielgenom leitet, an der sie die DNA schneidet. Der so entstandene Doppelstrangbruch wird durch ein zelleigenes Reparatursystem behoben. Die Reparatur kann entweder durch das fehleranfällige, nicht homologe Zusammenfügen der Enden erfolgen (NHEJ), wobei durch Fehler bei der Reparatur Mutationen entstehen und Gene inaktiviert werden können. Oder sie erfolgt über einen langsameren, gründlicheren Weg mithilfe einer Vorlage (Template). ${ }^{15}$ Mit dieser, durch homologe Template-Sequenzen (HDR) gezielt geleiteten Reparatur können gewünschte Sequenzen in Zielgene eingeführt werden. Die Kombination der Organoidtechnologie mit den diversen genetischen Editierungsmethoden schafft eine Fülle von Möglichkeiten, um Organoide genetisch zu verändern und Krankheitsmodelle zu entwickeln. Weitere Details und Beispiele einer Anwendung von Genetic Engineering mittels CRISPR/Cas9 in Organoiden werden im Folgenden diskutiert.

\subsubsection{Genetic Engineering von Organoiden aus adulten Stammzellen}

Adulte Stammzellen können direkt aus Geweben isoliert werden. Um genetisch modifizierte Organoide aus adulten Stammzellen zu erzeugen, kann man Stammzellen aus Tieren oder aus patienteneigenem Gewebe mit der gewünschten Genmutation gewinnen und diese dann durch Zellkultur in einer Laminin-Matrix unter Zugabe

15 Eine Vorlage kann etwa ein kleines Stück DNA mit einer erwünschten Sequenz sein, welches zusätzlich zu Cas-Protein und gRNA in die Zelle eingebracht wird und dort als Vorlage für die Reparatur dienen kann. So wird die gewünschte DNA-Sequenz in das Genom eingefügt. Der Prozess ist viel aufwendiger und daher langsamer, aber gründlicher als das bloße Verkleben der Enden. 
der benötigten Wachstumsfaktoren zu Organoiden weiterentwickeln (siehe Abbildung 2a). Allerdings kann es schwierig sein und viel Zeit und Geld kosten, eine mutierte Mauslinie zu erzeugen oder patientenspezifisches Gewebe zu erhalten. Insofern wäre es vorteilhaft, die Genexpression in Organoiden direkt erzeugen zu können. Solche eingebrachten Genmodifikationen wurden bereits in Organoiden des Dünndarmes (wie von einem der Autoren dieses Artikels 2012 veröffentlicht und erfolgreich zum Testen von Medikamenten verwendet), ${ }^{16}$ der Leber, des Pankreas und der Milchdrüsen durchgeführt.

2013 wurde von Schwank et al. ${ }^{17}$ in tierischen und auch in menschlichen Organoiden gezeigt, dass das CRISPR/Cas9-System transient durch Lipofektion in Organoide eingebracht und so Gene eingeschaltet und Mutationen repariert werden konnten. Das Forscherteam konnte mit dieser Methode bereits Mutationen in Organoiden von Patientinnen und Patienten mit zystischer Fibrose gezielt reparieren, und somit die Wirksamkeit der CRISPR/CAS9-Methode nicht nur in der Organoidforschung, sondern auch zur Korrektur von Gendefekten in der Krankheitsentstehung verdeutlichen. Zur Erforschung von Krebsentstehung können mithilfe dieser Methode Tumorsuppressorgene, die die Entstehung von Tumoren behindern, in Organoiden gezielt ausgeschaltet werden und die Krebsentwicklung im Detail studiert werden.

Ein weiterer Fokus der Geneditierung in Organoiden liegt darauf, die Wirkung von Onkogenen in der Tumorentwicklung zu untersuchen. So wurde etwa in Organoiden mit einem ausgeschalteten Tumorsuppressorgen untersucht, wie sich die Überexpression eines Tumorgens auswirkte. Während in Magen- und Pankreasorganoiden einfache Genmutationen ausreichten, um ein abnormales Wachstum hervorzurufen, war in Dickdarmorganoiden eine Kombination von mehreren Mutationen nötig. Dieses Multi-Hit-Modell der Krebsentstehung wurde mittlerweile von mehreren unabhängigen Studien in Darmorganoiden bestätigt (einen Überblick zu Organoiden in der Krebsforschung bietet Kretzschmar, Kap. 3.4).

Durch CRISPR/Cas9-Editierung konnten zudem Modelle für Krankheiten erzeugt werden, die zuvor in vitro nicht rekapituliert und somit nicht erforscht werden konnten, etwa bestimmte Vorstadien von Darmkrebs. Außerdem konnten mithilfe dieser neuen Technologie Darmorganoide für Medikamentenscreenings verwendet werden,

16 Koo, B. K. et al. (2012): Controlled gene expression in primary Lgr5 organoid cultures. In: Nature Methods 9(1): 81-83.

17 Schwank, G. et al. (2013): Functional repair of CFTR by CRISPR/Cas9 in intestinal stem cell organoids of cystic fibrosis patients. In: Cell Stem Cell 13(6): 653-658. 
Abbildung 2: Vergleich des Genetic Engineerings in Organoiden basierend auf adulten und pluripotenten Stammzellen
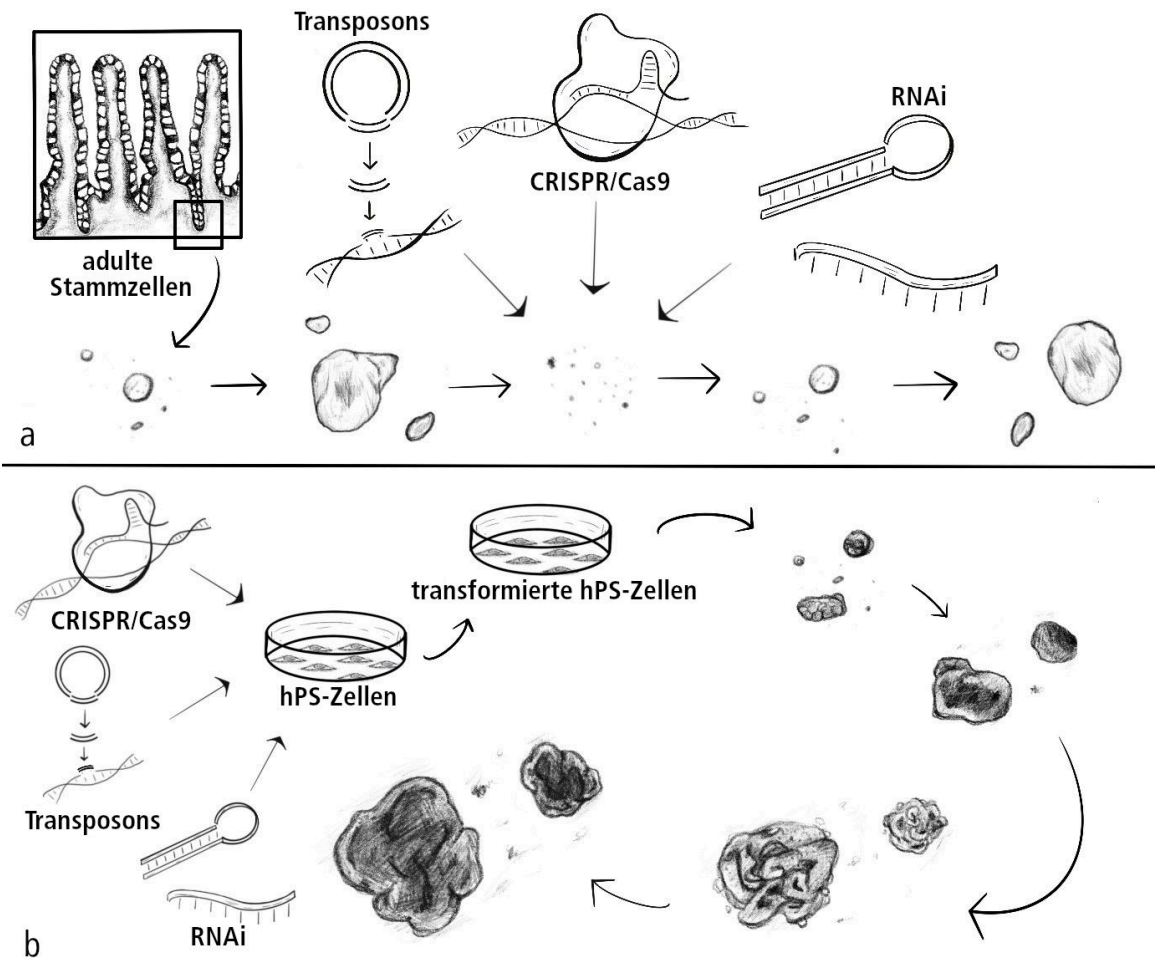

a) Um aus adulten Stammzellen gewonnene Organoide zu erzeugen, werden im Gewebe vorkommende Stammzellen isoliert und als Organoide kultiviert, damit sie sich unter In-vitro-Bedingungen stabilisieren können. Zur genetischen Modifikation werden die Organoide in einzelne Zellen aufgespalten, bevor das gewählte genetische Werkzeug eingeführt wird. Modifizierte Einzelzellen bilden im Anschluss wieder Organoide, die in Kultur gehalten oder zur Langzeitlagerung eingefroren werden können.

b) Aus pluripotenten Stammzellen (hPS-Zellen) gewonnene Organoide können vor der organoiden Differenzierung genetisch modifiziert werden. Geneditierungswerkzeuge können direkt in die hPS-Zellen eingeführt werden, bevor sie differenziert werden, um genetisch veränderte Organoide zu bilden. Nachdem sich die Organoide gebildet haben, ist es schwierig, sie wieder aufzuspalten, ohne dass sie ihre strukturelle Integrität und Funktion verlieren. Diese Abbildung stellt ein Beispiel für die verschiedenen Stadien der Bildung von Hirnorganoiden dar.

in denen die Auswirkungen verschiedener Genmutationen auf die Reaktion der jeweiligen Zellen auf bestimmte Medikamente untersucht wurden.

Um jedoch die Auswirkungen vieler gleichzeitig mithilfe von CRISPR/Cas9 ausgelöster Mutationen $\mathrm{zu}$ untersuchen (sogenanntes Multiplexing), müssen viele gRNAs 
kloniert und in die Zellen eingebracht werden. Eine Studie von Andersson-Rolf et al. ${ }^{18}$ entwickelte ein neues Werkzeug für die Expression von multiplexer gRNA in einem Konkatamer-Vektor, der die gleichzeitige Erzeugung von vier Gen-Knockouts (also Ausschaltungen von vier Genen gleichzeitig) ermöglicht. Aktuell wird an weiteren Verbesserungen des Multiplexings gearbeitet, da diese Methode vielversprechend für Screenings mit mehreren Zielgenen in zukünftigen Krankheits- und Krebsentstehungsstudien ist.

\subsubsection{Genetic Engineering von Organoiden aus pluripotenten Stammzellen}

Die Etablierung von geneditierten Organoiden aus pluripotenten Stammzellen ist vergleichsweise unkomplizierter als die aus adulten Stammzellen, da die Editierung direkt in den Stammzellen erfolgen kann, bevor aus ihnen Organoide hergestellt werden (siehe Abbildung 2b). Dieses Vorgehen ist effizienter, und erlaubt außerdem die Bildung von meist komplexeren Organoiden mit Zelltypen aus bis zu allen drei Keimblättern. Auf diese Weise wurden bislang beispielsweise geneditierte Hirnorganoide, Organoide des Verdauungstrakts und der Niere erzeugt.

Das Gehirn ist eines der komplexesten Organe im menschlichen Körper. Seine wissenschaftliche Untersuchung wurde bislang dadurch eingeschränkt, dass Hirngewebe vor allem durch ethische Bedenken schwer zugänglich ist. Mittlerweile konnten verschiedene Hirnregionen im Organoidmodell nachgebildet und für die Erforschung der Gehirnentwicklung genutzt werden (siehe hierzu ausführlich Tanaka/Park, Kap. 3.5, sowie für eine ethische Diskussion Schicktanz, Kap. 6). Da Hirnorganoide nur mittels pluripotenter Stammzellen gewonnen werden können, werden für die Züchtung oft humane induzierte pluripotente Stammzellen (hiPS-Zellen) verwendet, die aus verschiedensten Zelltypen, wie z. B. Hautzellen von Patientinnen und Patienten, erzeugt werden können, sowohl mit als auch ohne Geneditierung via CRISPR/Cas9. Aus diesen geneditierten hiPS-Zellen können Organoide erzeugt werden, die in weiterer Folge zur Erforschung der krankheitsauslösenden Mutationen bei den Patientinnen und Patienten dienen. Ein Nachteil dieser Methode besteht allerdings darin, dass alle Tochterzellen der Stammzellen, d. h. alle Zellen im Organoidverband, die Veränderung tragen. Daher kann die Interaktion zwischen gesunden und erkrankten Zellen nicht oder nur eingeschränkt untersucht werden, wobei diese in vivo jedoch eine wichtige Rolle bei der Entstehung von Krankheitssymptomen spielt. Einen Lösungsansatz bietet

18 Andersson-Rolf, A. et al. (2016): Simultaneous paralogue knockout using a CRISPR-concatemer in mouse small intestinal organoids. In: Developmental Biology 420: 271-277. 
eine neue Methode, bei der die gewünschten Mutationen mithilfe eines Transposons direkt in neuroepitheliale ${ }^{19}$ Zellen anstatt in pluripotente Stammzellen eingeführt werden. Die so entstandenen Organoide enthalten dann sowohl transformierte als auch nicht transformierte Zellen direkt nebeneinander, sodass sie mit der In-vivo-Situation vergleichbarer sind.

\subsubsection{Fazit}

Die Wahl des richtigen Delivery-Systems und der passenden Werkzeuge für Genetic Engineering ist komplex und bedarf sorgfältiger Abwägung, um die gewählte Methode der Genomeditierung an das jeweilige Organoidsystem und die $\mathrm{zu}$ beantwortende Forschungsfrage anzupassen. Dabei muss berücksichtigt werden, wann genau welche Veränderung in welchem Gewebe benötigt wird, ob diese transient oder permanent sein soll, und ob eine Züchtung aus adulten Stammzellen oder auch aus pluripotenten Stammzellen zielführender ist. Weitere Orientierung bieten folgende Fragen: Reicht eine - einfacher zu erzielende - Veränderung aller Zellen oder sollen nur bestimmte Zellen gezielt verändert werden? Soll ein komplettes Ausschalten bestimmter Gene (Knock-out) oder nur eine geringere Expression (Knock-down), durch eine Deletion oder Punktmutation, oder die Einfügung eines oder mehrerer Gene (Insertion, Knockin) erreicht werden, oder ist es strategisch besser, zu einem späteren Zeitpunkt einzugreifen und nicht das Gen, sondern das Protein oder dessen Zellstoffwechsel zu verändern? Auch wenn es verlockend ist, die neueste Methode einzusetzen oder möglichst hohe Komplexität zu erreichen, ist es meist effektiver und vor allem zeitsparender, die Technik auszuwählen, bei der man auf Erfahrung und Unterstützung im Forschungsumfeld zurückgreifen kann und die bestmöglich und gezielt auf die Forschungsfrage abgestimmt ist.

19 Neuroepitheliale Zellen sind Epithelzellen, die sich während der Embryonalentwicklung bilden (in der sogenannten Neuralplatte und dem Neuralrohr) und später zu Zellen des Nervensystems differenzieren. 